\title{
Synthesis and Characterization of Carbon Soot Particles Doped HPMC Polymer Composites
}

\author{
G.K. Gowtham, Vinayakprasanna N. Hegde, Simin Meshk, S.K. Sukrutha and \\ R. Somashekar
}

\begin{abstract}
Department of Studies in Physics, University of Mysore, Manasagangotri, Mysore - 570006, Karnataka, India
Abstract: Biodegradable Hydroxyl propyl Methyl cellulose based polymer composites of various concentrations were prepared by doping carbon particles of kerosene soot. These composites were synthesized by employing simple solution casting method, to study the physical properties of the same. The analytical studies like XRD, AC conductivity, Tensile strength, Acoustic impedance, AFM and FTIR spectroscopy were carried out to characterize these composites. From the results it is seen that, the amorphous carbon particle disturbs the semi crystalline nature of the polymer by getting interstitial into polymer network. Further, this results in the characteristic changes of other physical parameters like tensile strength and AC conductivity. We have made an attempt to establish a structure property relation of such changes in the physical properties with that of microstructural parameters derived from XRD.
\end{abstract}

Keywords: HPMC, Carbon Soot, XRD, Crystallite Size.

\section{INTRODUCTION}

There is an increase in research investigation on bio-degradable materials for various use in day to day life. In this context, Hydroxyl Propyl Methyl Cellulose (HPMC) is one such material. Hydroxyl propylmethyl cellulose (HPMC) belongs to the family of hydrophilic polymers, which when in contact with fluids swell and make a gel layer around dry core of the polymer matrix. Water-holding HPMC or its solution can absorb and hold moisture and it is easier to form smooth HPMC films which can be tough and elastic in its physical properties. These films are excellent oil resistant along with its antioxidant nature. HPMC is water soluble tasteless non-ionic derivative of cellulose, which is used as food additives, capsules and eye drops [1-3].

The particles of matter emitted from kerosene wick flames are hydrocarbons in origin, and are soot particles. These soot particles result from competition between soot formation and oxidation. Soot particles smaller than $300 \mathrm{~nm}$ are known to have a negative impact on health due to their physico-chemical properties. It is a known fact that carbon particles are amorphous and not so good conductor on its own. There is always an interesting enigma to know carbon nanoparticles behavior in a polymer matrix. Recently morphological characterization of carbon particles from soot without a catalyst precursor was reported [4] wherein they observe the presence of significant amount of carbon nanomaterial.

*Address correspondence to this author at the Department of Studies in Physics, University of Mysore, Mysore - 570006, Karnataka, India; Tel: +91821 2419608; E-mail: rs@physics.uni-mysore.ac.in
The main motive behind this research is to examine the changes in physico-mechanical properties of these polymer composites due to the addition of carbon particles and to study the interaction of soot model particles with the segment of polymer chain. In order to establish structure property relation in these composites insight to correlate the microstructural information with the physical properties.

\section{EXPERIMENT DETAILS}

\subsection{Sample Preparation}

HPMC polymer used in this research was obtained in powder form with an approximate molecular weight of 20,000 Daltons from Loba-Chemicals, Mumbai, India. The polymer films were prepared employing solution casting method [5]. Carbon soot particles were obtained by placing a flat glass plate over a kerosene lamp and scrapping off the soot collected from the surface of the glass into a container. These soot particles were added to 5\% HPMC solution in concentration of $0.01 \mathrm{~g}, 0.02 \mathrm{~g}, 0.03 \mathrm{~g}$ and $0.04 \mathrm{~g}$. Resulting in $\mathrm{C}_{1}, \mathrm{C}_{2}, \mathrm{C}_{3}$ and $\mathrm{C}_{4}$ samples which were dried under room temperature. A pure HPMC polymer film was also prepared for comparative studies.

\subsection{X-Ray Studies}

X-ray diffraction pattern of the sample was recorded using RigakuMiniFlex II Diffractometer with $\mathrm{Ni}$ filtered CuKa radiation of wavelength $1.5406 \AA$. The samples were scanned at a speed of $5^{\circ}$ per min with a step size of $0.02^{\circ}$ with a specification of $30 \mathrm{kV}$ and $15 \mathrm{~mA}$. In setting up the specimen and apparatus, co-planarity of the specimen surface with the specimen holder surface 
and setting of the specimen holder at the position of symmetric reflection geometry was ensured.

\subsection{FT-IR Studies}

The FTIR spectra was recorded in transmission mode with spectrophotometer model FTIR-4100 type A, having a resolution of $4 \mathrm{~cm}^{-1}$ in the wave number range $400-4000 \mathrm{~cm}^{-1}$. The nature of chemical bonds in polymers can be studied through the characterization of the vibration modes by infrared spectroscopy [6].

\subsection{Acoustic Impedance}

The ease with which an ultrasonic pulse can travel through a material depends on a property of the material called Acoustic impedance (Z). Digital Ultrasonic Pulse Echo Velocity Meter with silicon grease as couplant is used to determine Acoustic impedance of the polymer samples in both Longitudinal and Shear modes.

\subsection{AC Conductivity}

AC Conductivity measurements for the HPMC polymer films of different carbon concentrations were measured using HIOKI 3532-50 LCR HiTESTER, the test frequency was set from $50 \mathrm{~Hz}$ to $5 \mathrm{MHz}$ under room temperature.

\subsection{Tensile Strength}

The mechanical properties of the polymer samples were recorded using Universal Testing Machine (Lloyd Instruments, UK, $5 \mathrm{kN}$ load cell). Each sample was tested at a specification of $50 \mathrm{~mm}$ gauge length, with the crosshead speed of $20 \mathrm{~mm} / \mathrm{min}$ at room temperature. A model LRX plus was used to determine the maximum tensile strength, maximum percentage elongation at break and elastic modulus.

\subsection{AFM Studies}

The surface morphology of polymer samples were carried out using NanosurfeasyScan2, Atomic Force Microscope (AFM-Switzerland) in contact mode with a scanner size of $12 \mu \mathrm{m} \times 12 \mu \mathrm{m}$.

\section{RESULTS AND DISCUSSION}

X-ray diffraction pattern obtained for the polymer composites are shown in Figure 1. It can be seen that the diffraction patterns obtained for the doped polymer composites are similar to that of the pure HPMC film with change in its intensity i.e., the addition of carbon

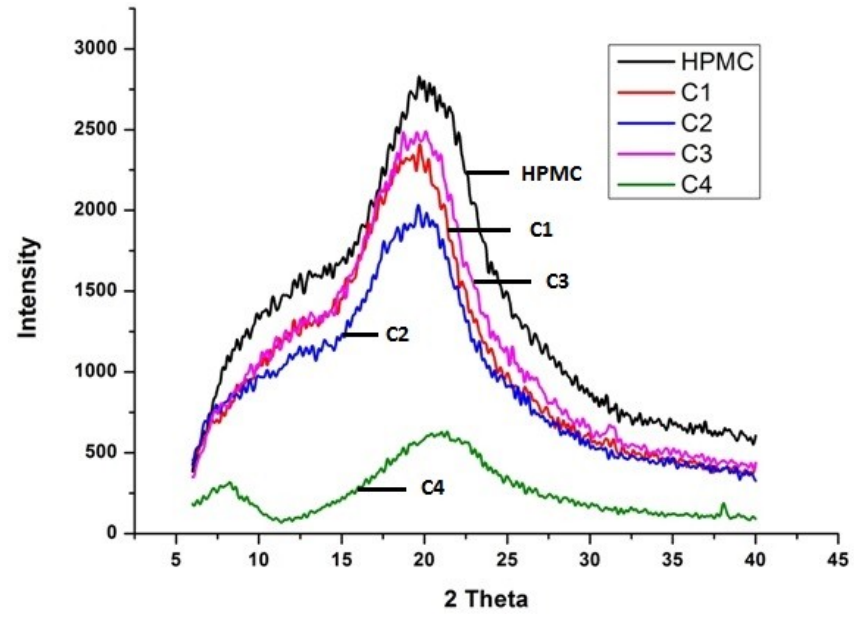

Figure 1: XRD pattern of HPMC and Carbon particles doped in HPMC.

particles to the HPMC polymer do not affect the parent polymer matrix significantly but makes it more amorphous resulting in less intense peaks compared to pure HPMC. This is further supported by the crystallinity values calculated by Williamson-Hall plot method [7]. W-H plot considers both limited size of the crystals and the presence of crystallographic distortions which leads to Lorentzian intensity distributions. The slope of the $\mathrm{W}-\mathrm{H}$ plot represents the average strain in the crystal, whereas intercept with the $y$-axis gives the crystallite size. The Williamson-Hall relation is given by

$$
\beta \frac{\cos \theta}{\lambda}=\frac{1}{D}+4 \varepsilon \frac{\sin \theta}{\lambda}
$$

Where ' $\beta$ ' is the full width at half maximum (FWHM) of the peak measured in radians, ' $D$ ' is the average crystallite size, and ' $\varepsilon$ ' is the average lattice strain [8]. The calculated microstructural parameters are tabulated in Table 1. But further, for higher concentrations, carbon particles dominate in the polymer matrix and results in higher crystallinity values. Though the crystallite size decreases for the first two concentration it increases as the concentration of carbon particle increases, this is because at higher

Table 1: Microstructural Parameters of HPMC and Carbon Particles Doped in HPMC

\begin{tabular}{|c|c|c|}
\hline Sample & Strain (\%) & Crystallite Size (A) \\
\hline \hline HPMC & 1.4 & 13.3 \\
\hline $\mathrm{C}_{1}$ & 6.9 & 10.4 \\
\hline $\mathrm{C}_{2}$ & 2.3 & 8.9 \\
\hline $\mathrm{C}_{3}$ & 2.0 & 11.3 \\
\hline $\mathrm{C}_{4}$ & 1.1 & 14.2 \\
\hline
\end{tabular}


concentration carbon soot particles try to dominate in the matrix and thus camouflage the structure of parent polymer. The crystallite size values obtained for composites at higher concentration is a resultant of both the polymer and carbon particles.

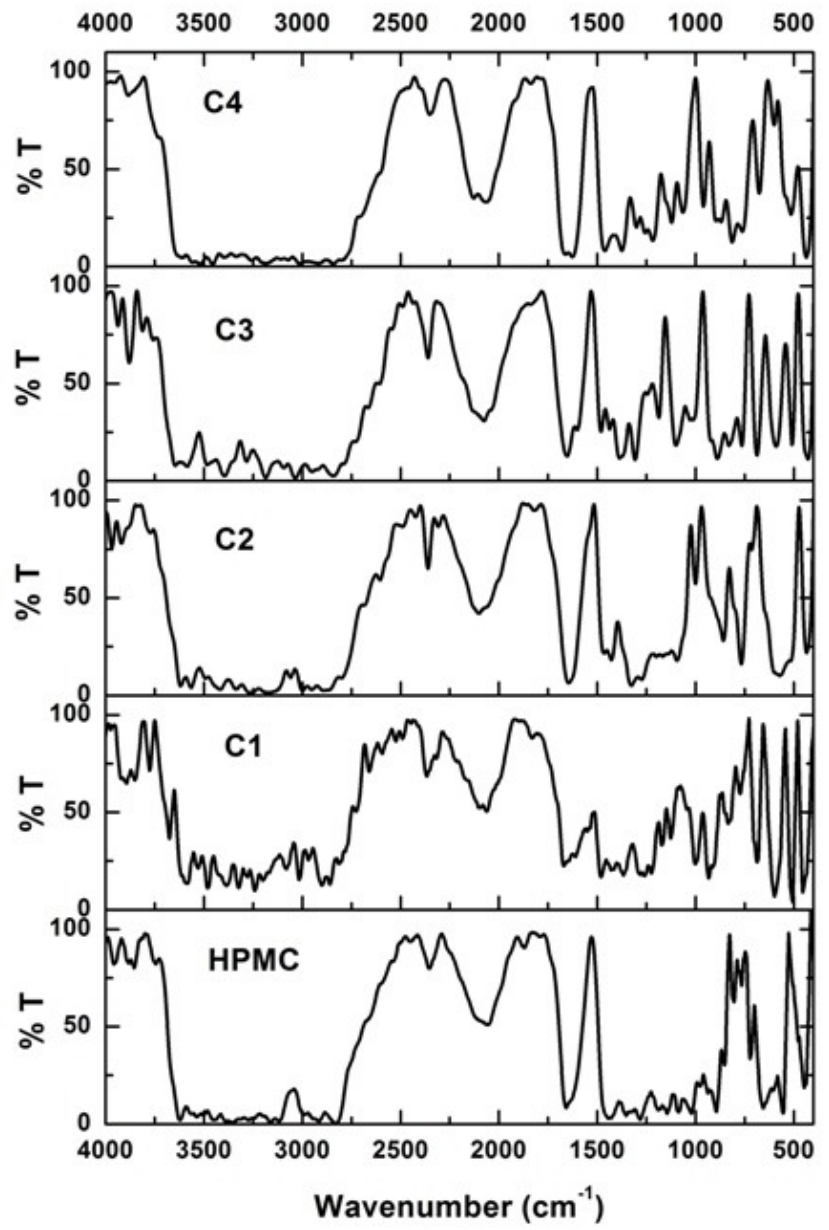

Figure 2: FT-IR spectra of Pure and Carbon soot doped HPMC films respectively.

FTIR absorbance spectra for pure and the carbon doped films of HPMC polymer samples are shown in Figure 2. In the IR spectra we have considered a few prominent bands around $2800 \mathrm{~cm}^{-1}$ which corresponds to $C-H$ stretch. Around $1650 \mathrm{~cm}^{-1}$ the bands corresponds to stretching of $-C=C-$ groups. For pure $\mathrm{HPMC}$, the fundamental and first overtone transition of $-C=C-$ bonds are centered at $1657 \mathrm{~cm}^{-1}$ and $2827 \mathrm{~cm}^{-1}$ respectively. The equilibrium Vibration frequency of the chain about this bond is calculated using the relations $[9,10]$. FTIR results tabulated in Table 2 do correlate with that of XRD which are indicated by the changes in anharmonicity and force constant. Initially force constant increases with increase in carbon particles up to $0.03 \%$ and thereafter it decreases. This is due to changes in polymer network in the neighbourhood of carbon particles. But, at the outset it is seen that both the anharmonicity and force constant varies about a constant mean and do not exceed pristine values. This confirms that the carbon particles in the network is physically interstitial and do not break the chemical chains of the parent matrix. Thus, the concentration of carbon particles in the matrix results in the variation of these values, and thereby is in accordance with the results of XRD.

Acoustic Impedance ' $Z$ ' is calculated using the relation $\boldsymbol{Z}=\boldsymbol{\rho} \times \boldsymbol{V}$, where ' $\boldsymbol{\rho}$ ' is density and ' $\mathbf{V}$ ' is the velocity. Both longitudinal and shear modulus of polymer composite decreases with increase in carbonconcentration as shown in Table 3. This indicates that there is considerable breaking of intermolecular interactions in these systems [11, 12].

After obtaining transconductance (G) from the instrument, AC conductivity $(\boldsymbol{\sigma})$ for the polymer composites were obtained by the relation,

$$
\sigma=\frac{(t \times G)}{A}
$$

Where ' $\mathrm{t}$ ' is the thickness and ' $\mathrm{A}$ ' is the area of the sample

Figure 3 shows the plot of conductivity as a function of log Frequency. From the results obtained, it is found

Table 2: IR Data for Pure and Carbon Soot Doped HPMC Films

\begin{tabular}{|c|c|c|c|c|}
\hline Sample & $\begin{array}{c}\text { Anharmonicity } \\
\text { Constant } \\
\left(\boldsymbol{\chi}_{\left.\mathrm{e} \times \mathbf{1 0}^{-2}\right)}\right.\end{array}$ & $\begin{array}{c}\text { Equilibrium } \\
\text { Vibration } \\
\left(\bar{v}_{e}\right)\end{array}$ & $\begin{array}{c}\text { Zero point Energy }\left(\varepsilon_{\mathbf{o}}\right) \\
\left(\mathbf{c m} \mathbf{- 1}^{-1}\right)\end{array}$ & $\begin{array}{c}\text { Force } \\
\text { Constant } \\
(\mathbf{N})\end{array}$ \\
\hline \hline $\mathrm{NPMC}$ & 11.4 & 2145.4 & 1011.7 & 256.7 \\
\hline $\mathrm{C}_{1}$ & 10.5 & 2113.6 & 1001.5 & 249.1 \\
\hline $\mathrm{C}_{2}$ & 9.8 & 2046.1 & 0973.1 & 233.5 \\
\hline $\mathrm{C}_{3}$ & 10.9 & 2113.6 & 0999.3 & 249.1 \\
\hline $\mathrm{C}_{4}$ & 10.1 & 2035.5 & 0966.6 & 239.0 \\
\hline
\end{tabular}


Table 3: Longitudinal and Shearmodulus

\begin{tabular}{|c|c|c|c|c|c|}
\hline Sample & $\begin{array}{c}V_{1} \\
(m / s)\end{array}$ & $\begin{array}{c}V_{s} \\
(m / s)\end{array}$ & $\begin{array}{c}\rho \\
\left(\mathrm{kg} / \mathrm{m}^{3}\right)\end{array}$ & $Z_{1}\left({ }^{*} 10^{5}\right)\left(\mathrm{kg} / \mathrm{m}^{2} \mathrm{~s}\right)$ & $Z_{\mathrm{s}}\left({ }^{*} 10^{5}\right)\left(\mathrm{kg} / \mathrm{m}^{2} \mathrm{~s}\right)$ \\
\hline HPMC & 280 & 350 & 1438.4 & 4.3 & 5.0 \\
\hline $\mathrm{C}_{1}$ & 340 & 425 & 1357.0 & 4.6 & 5.8 \\
\hline $\mathrm{C}_{2}$ & 320 & 400 & 1352.1 & 4.0 & 5.0 \\
\hline $\mathrm{C}_{3}$ & 300 & 375 & 1283.4 & 3.9 & 4.8 \\
\hline $\mathrm{C}_{4}$ & 280 & 350 & 1305.3 & 3.7 & 4.6 \\
\hline
\end{tabular}

$\mathbf{V}_{\mathbf{l}}$ : Longitudinal Velocity, Vs: Shear Velocity, $\mathbf{\rho}$ : Density, $\mathbf{Z}_{\mathbf{l}}$ : Acoustic Impedance (Longitudinal), Zs: Acoustic Impedance (Shear).

that, for each concentration conductivity increases with increase in frequency. But with increase in carbon concentration we can observe an exponential decrease in conductivity of the samples. This is due to the fact that carbon nanoparticles in the polymer network behaves to be more dielectric [13] and hence there is a considerable decrease in the conductivity values. This behavior of the polymer composites is represented by a bezier fit of conductivity versus concentration, for a frequency of $10 \mathrm{kHz}$ as shown in Figure 4.

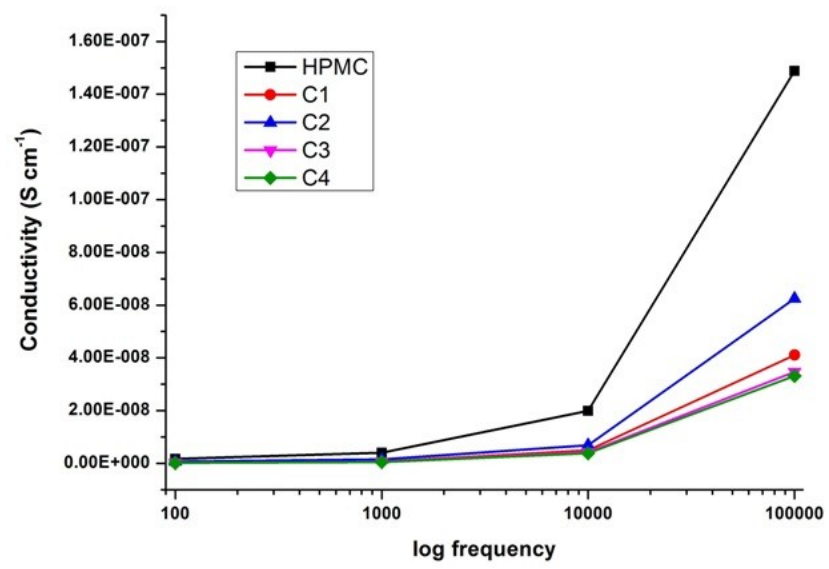

Figure 3: Variation of AC conductivity with log frequency.

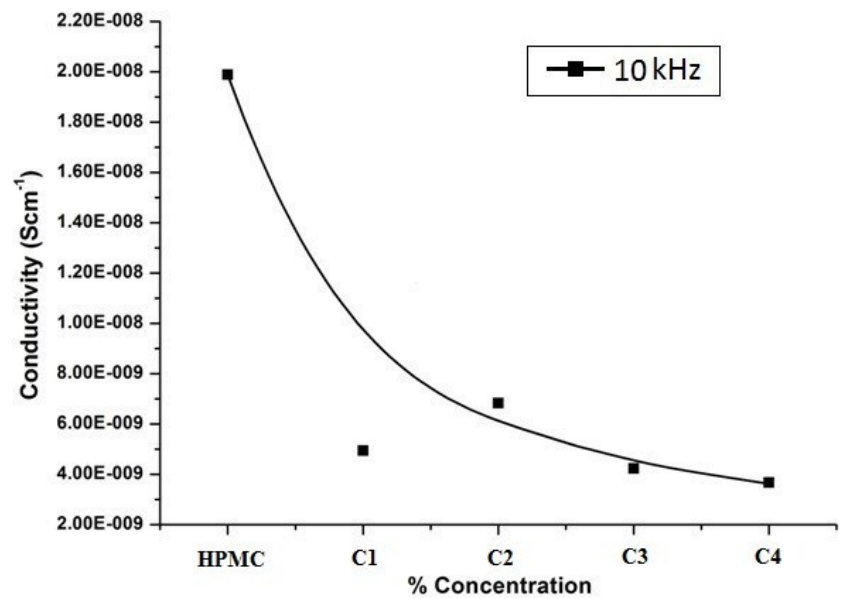

Figure 4: Variation of AC conductivity with Carbon Concentrations.
Tensile strength, percentage of elongation and elastic modulus are parameters that are related with the mechanical properties of the sample and their chemical structures. Figure $\mathbf{5}$ shows stress-strain curve for the samples. The values of Tensile strength, Young's modulus and percentage of elongation for HPMC polymer samples with carbon concentrations are presented in Table 4. The tensile strength of the polymer composites vary with carbon concentration, which is found to be maximum for pure HPMC (43.14 $\mathrm{MPa}$ ) and minimum for the sample $\mathrm{C}_{4}$. Stress-Strain curve for pure HPMC indicates its high Tensile strength and modulus with higher range of elastic limit. It has

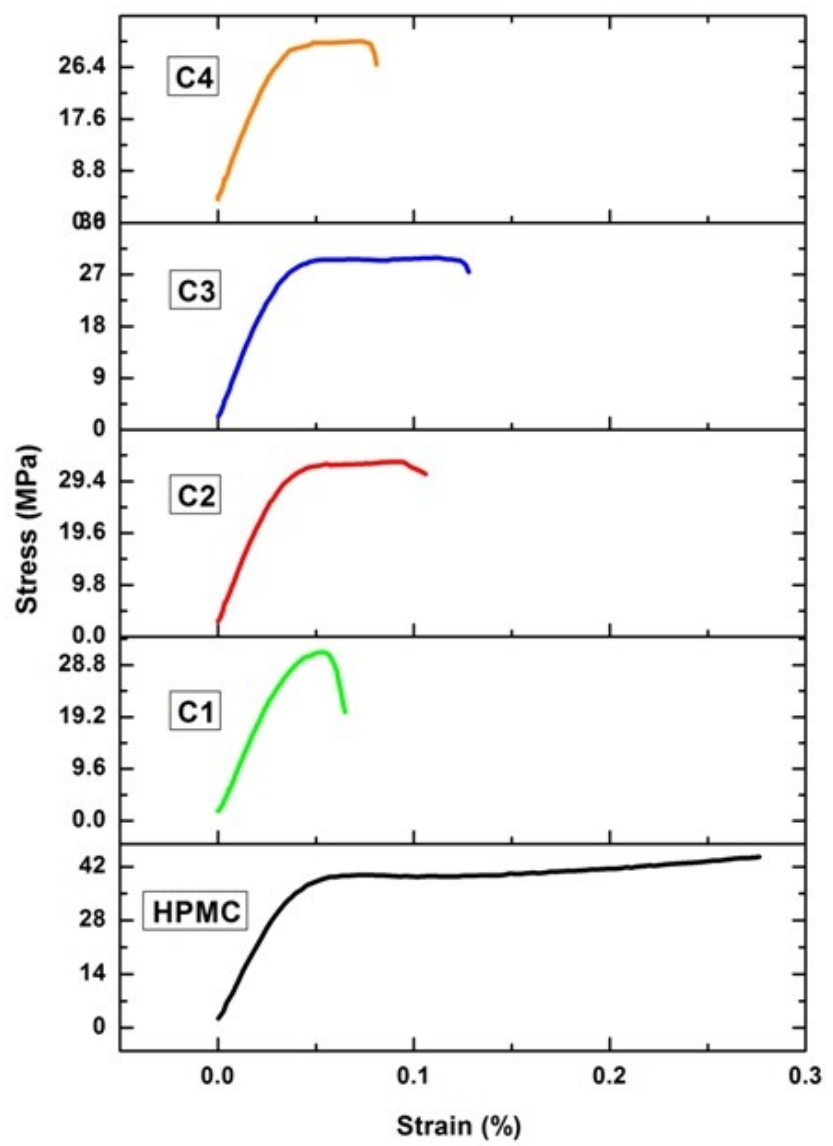

Figure 5: Stress vs Strain Curve. 
Table 4: Mechanical Properties of Pure and Carbon Soot Doped HPMC Polymer Composites

\begin{tabular}{|c|c|c|c|c|}
\hline Sample & \% Elongation Maximum & Tensile Strength (MPa) & Young's Modulus (MPa) & $\begin{array}{c}\text { Elongation at Fracture } \\
\text { (mm) }\end{array}$ \\
\hline \hline HPMC & 17.8 & 43.1 & 1278.7 & 5.9 \\
\hline $\mathrm{C}_{1}$ & 5.4 & 31.2 & 949.3 & 2.0 \\
\hline $\mathrm{C}_{2}$ & 11.0 & 37.2 & 1169.4 & 3.7 \\
\hline $\mathrm{C}_{3}$ & 12.2 & 29.9 & 1082.1 & 4.2 \\
\hline $\mathrm{C}_{4}$ & 6.6 & 34.1 & 1147.0 & 2.3 \\
\hline
\end{tabular}

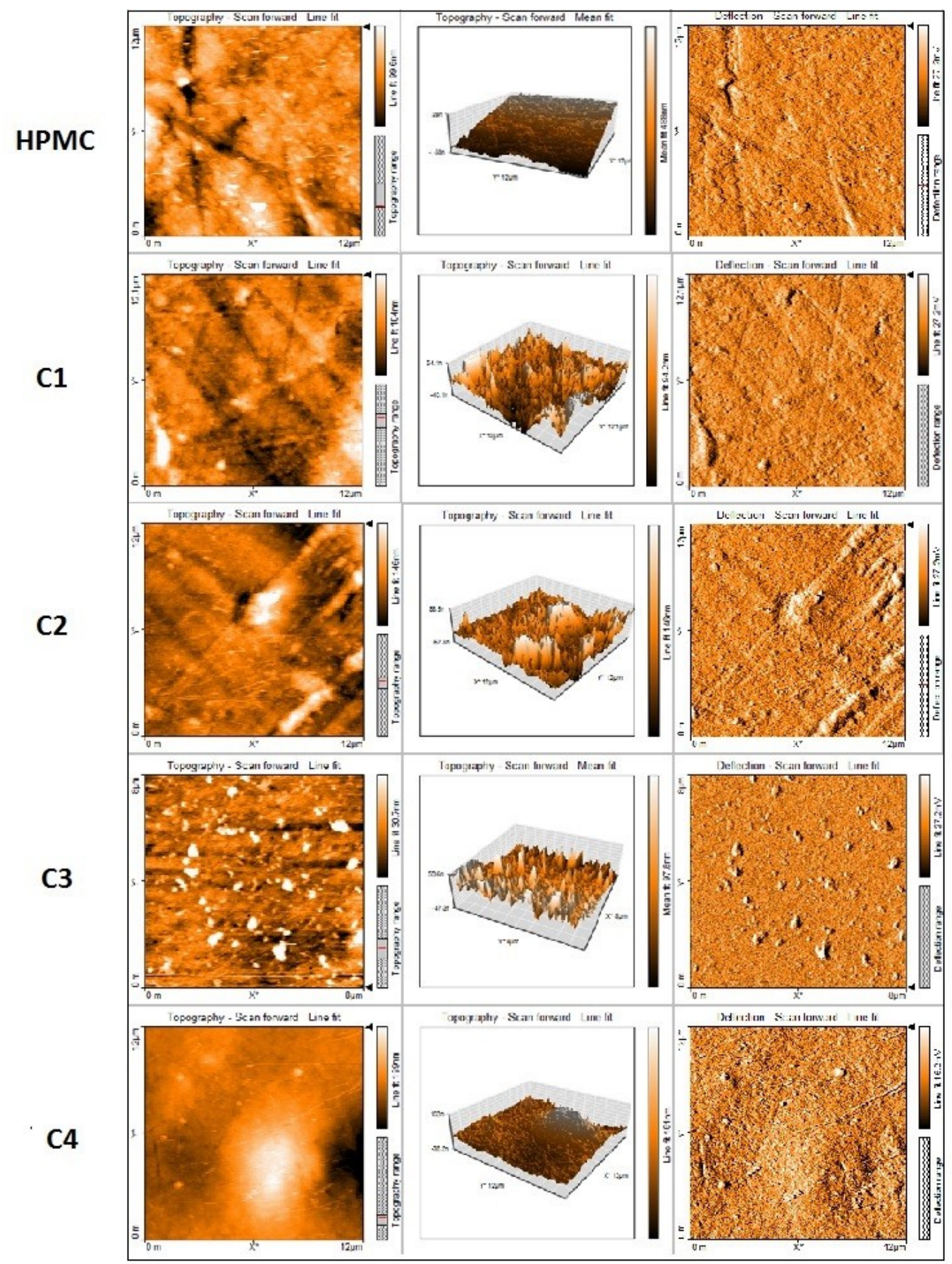

Figure 6: AFM Images of Pure HPMC and carbon doped HPMC concentrations. 
Table 5: AFM Analysis of HPMC- Carbon Particle Films

\begin{tabular}{|c|c|c|c|c|c|c|}
\hline Sample & $\mathbf{R a}(\mathbf{n m})$ & $\mathbf{R q}(\mathbf{n m})$ & $\mathbf{R y}(\mathbf{n m})$ & $\mathbf{S a}(\mathbf{n m})$ & $\mathbf{S q}(\mathbf{n m})$ & $\mathbf{S y}(\mathbf{n m})$ \\
\hline \hline $\mathrm{HPMC}$ & $13.0 \pm 3.0$ & $17.4 \pm 2.7$ & $87.4 \pm 27.5$ & $12.4 \pm 3.5$ & $16.1 \pm 4.6$ & $129.3 \pm 42.1$ \\
\hline $\mathrm{C}_{1}$ & $11.6 \pm 2.0$ & $14.2 \pm 2.4$ & $67.4 \pm 14.7$ & $13.9 \pm 4.8$ & $18.0 \pm 6.0$ & $122.5 \pm 23.7$ \\
\hline $\mathrm{C}_{2}$ & $13.2 \pm 1.7$ & $17.1 \pm 5.0$ & $92.8 \pm 18.5$ & $16.9 \pm 8.6$ & $21.6 \pm 11.00$ & $146.1 \pm 64.8$ \\
\hline $\mathrm{C}_{3}$ & $15.7 \pm 2.1$ & $19.0 \pm 3.6$ & $76.2 \pm 20.6$ & $12.2 \pm 5.2$ & $18.2 \pm 1.5$ & $124.5 \pm 33.6$ \\
\hline $\mathrm{C}_{4}$ & $23.2 \pm 17.0$ & $27.3 \pm 19.8$ & $114.5 \pm 80.1$ & $20.3 \pm 16.6$ & $25.5 \pm 19.3$ & $149.0 \pm 87.1$ \\
\hline
\end{tabular}

Ra,Sa: average values of line and surface roughness, Rq,Sq: root mean square value of line and surface roughness, Ry,Sy: maximum value of line and surface roughness.

high flexibility when compared to other films. All films have same initial modulus, however the sample $\mathrm{C}_{4}$ has a little high initial modulus than others. Only pure HPMC has longer elastic limit and plastic region when compared to othersamples. This shows addition of carbon particles have affected the plasticity of HPMC polymer matrix.

Figure 6 shows the topography, 3D AFM and deflection images of the polymer composites, from which the average sample roughness value is obtained. Sample roughness corresponds to the particle size distribution and sample nature [14]. The line roughness parameters $\left(R_{a}, R_{q}\right.$ and $\left.R_{y}\right)$ and surface roughness parameters $\left(S_{a}, S_{q}\right.$ and $\left.S_{y}\right)$ are physical scales which describes the roughness degree of the samples which are presented in Table 5. In most of the regions the sample exhibit irregular particle dimension and have high $R_{q}$ and RMS roughness value, this is maximum for the $\mathrm{C}_{4}$ sample. From the resultsobtained, a decrease inmechanical properties and an increase in surface roughness of the polymer composites can be observed.

\section{CONCLUSION}

With an interest to study the properties of carbon soot as an effective dopant in a polymer matrix, an attempt has been made to characterize polymer composites by various analytical measurements. The main expectation behind was in its conducting property, which exhibited contradictory results. Even though characterizations like XRD, FTIR, Acoustic Impedance and AFM show some characteristic behaviour with the concentration of carbon particles,the overall conductivity of the composites have not shown a positive change. Carbon soot particle in the matrix arrange themselves in the form of small crystallite like structures and these clusters behave to be a hindrance for conductivity. Soot particles being more dielectric in nature, makes these polymer composites to be nonconductive. This indicatesthat the addition of carbon soot to the polymer exterminates the semiconducting property of the host matrix. Thus, the use of carbon soot particles as conducting dopant is not recommended.

\section{REFERENCES}

[1] Siepmann J, Peppas NA. Modelling of drug release from delivery systems based on hydroxypropyl methylcellulose (HPMC). Adv Drug Deliv Rev 2001; 48: 139-57.

http://dx.doi.org/10.1016/S0169-409X(01)00112-0

[2] Phaechamud T. Variables Influencing Drug release from layered matrix system comprising Hydroxypropyl Methylcellulose. AAPS Pharm Sci Tech 2008. http://dx.doi.org/10.1208/s12249-008-9085-1

[3] Talukdar MM, Michoel A, Rombout P, Kinget R. Comparative study on Xanthan gum and Hydroxypropyl methyl cellulose as matrices for controlled drug delivery. I.Compaction and In vitro drug release behaviour. Int J Pharm1996; 129: 233-41.

[4] Ntaote David Shooto, Ezekiel Dixon Dikio. Synthesis and characterization of Diesel, Kerosene and Candle wax soot's. Int J Electrochem Sci 2012; 7: 4335-44.

[5] Tawansi A, Zidan HM, Oraby AH, Dorgham ME. Short-rangeorder spin clusters in one-dimensional Ising-like antiferromagnetic $\mathrm{CoBr}_{2}$-filled PVA films: a study of physical properties. J Phys D: Appl Phys 1998. http://dx.doi.org/10.1088/0022-3727/31/24/005

[6] Davenas J, Xu XL, Boiteux G, Sage D. Relation between structure and electronic properties of ion irradiated polymers. Nucl Instr Meth B 1989; 39: 754-63.

http://dx.doi.org/10.1016/0168-583X(89)90891-4

[7] Williamson GK, Hall WH. X-Ray line broadening from filed aluminium and wolform. Acta Mettalurgica 1953; 1: 22-31. http://dx.doi.org/10.1016/0001-6160(53)90006-6

[8] Urs TGK, Dasaiah M, Somashekar R. Studies on structural and conducting properties of goethite nanoparticles doped HPMC polymer films. J Polym 2014. http://dx.doi.org/10.1155/2014/201464

[9] Aruldhas G. Molecular Structure and Spectroscopy, 2nd ed Prentice-Hall of India, New Delhi, 2004.

[10] Sangappa AS, Demappa T, Sanjeev G, Parameswara P, Somashekar R. Spectroscopic and thermal studies of $8 \mathrm{MeV}$ electron beam irradiated HPMC films. 2009; 267: 2385-9.

[11] Bajram Z, Janine A. Couplants and their influence on $A E$ sensor sensitivity Pete Theobald, National Physical Laboratory. J Acoustic Emission 2008; 26: 91-7.

[12] Guofeng B, Fusheng S, Jun Y, Chengguang Z. Elastic wave scattering by periodic cavities in backing material for ultrasound transducers. Available from http://medyna2013. sciencesconf.org/13237/document. 
[13] Yujie H, Robert CS, Keith NJ, Linda S. Schadler Rensselaer Some mechanistic understanding of the impulse strength of nanocomposites. Available from http://homepages.rpi.edu/ $\sim$ nelsoj/CEIDP2006_4.doc.
[14] Soundappan T, Rong FY, Shen-Ming C. Palladium particles based nano films electrochemical fabrication, characterization and applications. Int J Electrochem Sci 2011; 6: 4537-52.

Received on 08-06-2015

Accepted on 01-07-2015

Published on 28-07-2015

DOI: http://dx.doi.org/10.6000/1929-5995.2015.04.02.1 